Journal of the Operations Research

Society of Japan

2003 , Vol. 46 , No. $4,503-522$

\title{
REGENERATIVE CYCLE METHOD IN M/G/1 WITH MANY VACATION RULES
}

\author{
Toshinao Nakatsuka \\ Tokyo Metropolitan University
}

(Received October 22, 2002; Revised April 10, 2003)

\begin{abstract}
This paper gives a new method to derive the time average distribution of queue length in the model $M / G / 1$ with many vacation rules. We extract many kinds of regenerative cycles of the well-known regenerative processes from the queue length process of the model by using the method shown by Fuhrmann and Cooper. The time average of the original model is represented by the combination of the time averages of such regenerative processes. We find this combination in various models, e.g., the model with setup time, the special decrementing service system, pure limited service system, feedback system and so on. We show a recursive calculation formula for moments.
\end{abstract}

Keywords: Queue, regenerative cycle method, M/G/1, vacation, pure limited service system

\section{Introduction}

The $M / G / 1$ is the fundamental queueing model and its many properties have been discovered. The variants of $M / G / 1$ are also, sometimes more, important in real application to many fields of operations research. These variants give rise to many problems which we must solve. This paper was stimulated from the study of the queue length of the $M / G / 1$ with server vacations. In the setting of early authors the vacation begins only when there is no customer in the system(e.g., [3]). They(see Takagi's book[20] or Doshi's servey[5]) studied the model with multiple vacation or the model with $N$-policy, and derived the probability generating function(PGF) of the queue length at the departure epoch or at an arbitrary time as the decomposition form. Afterwards some authors considered the case that the server takes a vacation even when there is a customer in the system. Particularly Fuhrmann and Cooper[7] obtained the generalized decomposition theorem about queue length in the model with one type of vacation. Shanthikumar[16] pointed out that such decomposition holds for vacations other than an ordinary vacation. However their technique has not prevailed. For example, Takagi([20] p.202) introduced most results in the gated service system or other important models by using other technique. Wortman and Disney[23] used the Markov renewal process embedded in the model. One reason is thought to be that the generalized decomposition theorem is available for the model with only one type of vacation. Therefore, the applicable models are limited.

When we consider the real application, the analysis of the model with several vacation rules is necessary. In such model their decomposition theorem is not useful. Really, if we regard the idle period in $M / G / 1$ as a vacation, it is essentially different with the ordinary vacation which is independent of the arrival epochs. So it is difficult to deal with these vacations all together as one type of vacation. The model with two or three types of vacation has been discussed individually, e.g., gated service systern with multiple vacation([17]), 
threshold policy model of [26], etc. This paper considers Fuhrmann and Cooper's technique in such model and obtains the time average PGF of the queue length $y_{t}$ for some variants of $M / G / 1$.

Fuhrmann and Cooper defined the vacation customers and their offsprings whom one vacation generates. From any sample path of the model they extracted the time domain consisting of one vacation period and service periods of its customers and offsprings. This paper regards their time domain as one cycle of the regenerative process. Therefore if the model has many vacation rules, it generates many types of such regenerative cycles. We represent the time average PGF of $y_{t}$ by the combination of the PGF's of the regenerative processes which the vacations of this model generate.

Conversely speaking, we can make new model by combining the regenerative processes. As the fundamental regenerative processes, this paper chooses $M / G / 1$ with $N$-policy and $M / G / 1$ with multiple vacation. The former is the regenerative process generated by $N$ policy vacation and the latter is the regenerative process generated by the ordinary vacation. Successive occurrence of the regenerative cycles of these two kinds of regenerative processes generates many interesting variants of $M / G / 1$. Since the time average PGF's of these two models are well known, we can calculate PGF of the variant model through the combination of the PGF's of these two fundamental models. We illustrate our technique in many examples, e.g., model with setup time, special decrementing service system, pure limited service system, feedback system and so on. Moreover Theorem 4.1 which is our main theorem is not restricted only to the combination of the fundamental regenerative cycles. If we obtain the PGF's of complicated regenerative processes, we can obtain the PGF of more complicated model by combining these regenerative processes. However, since one purpose of this paper is to show the possibility of easy calculation, we do not deal with the extremely entangled example.

In order to consolidate the foundations, we must discuss strictly and clarify the condition. This paper uses the $M / G / 1$ with multiple vacation as an important fundamental regenerative process. The cycle length between regeneration points in this model has the lattice distribution for special distributions of service and vacation and for a special initial condition in spite of the Poisson arrival, so that it has not the limiting distribution for such initial state. Moreover the stability of the model is not directly related with Theorem 4.1. Therefore we focus our attention to the time average.

The technique of this paper derives the time average PGF of the queue length for many models. We can obtain probabilities and moments of its probability measure by differentiating it. The representation of the combination is also useful for this calculation. However the differentiation of some fundamental PGF's is necessary and it is laborious if we try it directly. In last section we improve the classical recursive calculation method.

\section{2. $M / G / 1$ With Vacations}

\subsection{The customers generated by a vacation}

Before discussing the general theory in sections 3 and 4 , this section defines a time domain in a queueing system which consists of the vacation interval and the service times of customers generated by its vacation. This time domain is the typical example of the time domain discussed in the general theory. Moreover, we use it as a fundamental regenerative cycle in later sections. Every queueing system in our examples has a single server. Our system starts at the epoch zero with an idle state. Let $y_{t}$ be the number of customers in the system at a time epoch $t \in[0, \infty)$. The server sometimes takes vacations. In this paper we regard 
an idle period as a vacation, too. So any epoch on the total time domain $[0, \infty)$ belongs to a service period on which the server is working or to a vacation period on which the server is not working. The customers arrive according to Poisson process with constant intensity $\lambda$. On the other hand the service times are i.i.d. with the distribution function $B(x)$ and they are independent of the arrival process. Let $b$ and $B^{*}(s)$ be the mean and the Laplace Stieltjes transform(LST) of $B(x)$ respectively. We put $\rho=\lambda b$. The service discipline is the nonpreemptive LIFO(Last In, First Out). In many cases the stochastic behavior of $y_{t}$ is identical among nonpreemptive LIFO, FIFO, service in random order or other similar service disciplines, so that we can apply our results to these cases.

The various kinds of vacation rules are possible as is shown in $[16,6]$. Particularly with respect to the arrivals during a vacation we can consider balking, reneging and other rules on the vacation. However the examples in this paper use the only following two kinds of vacations.

1. The ordinary vacation. Once this vacation begins, its length of vacation period is determined independently of the arrival process and service times. All customers who arrive during the vacation period continue to stay in the system until the end of this period.

2. $N$-policy vacation. The $N$ customers arrive during this vacation. This vacation ends when the last customer among these $N$ customers arrives. As the special case, the idle time interval in the ordinary $M / G / 1$ is the vacation period with 1 -policy.

We denote the $k$ th vacation after the starting epoch zero by the notation $V^{k}$. In [7] the customers who arrive during the vacation $V^{k}$ are called the vacation customers and their set is denoted by $I_{0}^{k}$. To define strictly, we assume that the customer who happens to arrive just at the beginning of $V^{k}$ does not belong to $I_{0}^{k}$ and the customer who happens to arrive just at the end of $V^{k}$ belongs to $I_{0}^{k}$. That is, the vacation period is the half open interval with the form $(a, b]$, if it is not interrupted. The important example of the former case is the model with setup time and the important example of the latter case is the $N$-policy rule.

The customers who arrive while the members of $I_{i-1}^{k}$ are being served are called the $i$ th generation offsprings and their set is denoted by $I_{i}^{k}$. We put $G_{k} \equiv \cup_{i=0}^{\infty} I_{i}^{k}$. Two different vacations do not duplicate, so that $G_{i} \cap G_{j}$ is the null set for $i \neq j$.

In section 4 we define the time domain $J_{k}(\subset(0, \infty))$ generally. As its typical example, this section considers the union of the time interval of $V^{k}$ and the service periods on which the customers in $G_{k}$ are served. If $J_{k}$ is so, in many variants of $M / G / 1$ we often find that a certain vacation $V^{k+1}$ starts before $J_{k}$ ends. In this case the remaining customers in $G_{k}$ wait until $J_{k}$ resumes. We should note that the $J_{k}$ resumes after the completion of $J_{k+1}$ because of LIFO discipline. Under the assumption of Poisson arrival the probability behavior of the queue length on the $J_{k}$ is not influenced by the interruption of $J_{k+1}$. If this is guaranteed, we can consider the case that the vacation begins in the middle of the service. Let $l_{k}$ be the number of customers staying at the beginning of $V^{k}$. Since these customers do not belong to $G_{k}$ and are served after $J_{k}$, the probability structure of $y_{t}-l_{k}$ on $J_{k}$ is determined only by the arrivals on it and services of customers in $G_{k}$. So we call $y_{t}-l_{k}$ the regenerative cycle on $J_{k}$. If $J_{k}$ is generated by a certain vacation rule, the regeneration cycle on it has the probability structure which is peculiar to this vacation rule. Since we consider the model with many vacation rules, we classify such probability structure by the type number $\xi_{k}$.

\subsection{Examples}

The following two examples have both 1-policy and an ordinary vacation. In Example 2.1 one $J_{k}$ is interrupted by only one other vacation. In Example 2.2 one $J_{k}$ is interrupted by several other vacations. 
Example 2.1. Consider the $M / G / 1$ with setup time. Assume $\rho<1$. When there is no customer in the system, the server waits for next customer. When a customer arrives at the idle system, the server takes a setup time. After the completion of the setup time he continues to work until there is no customer in the system. Assume that the setup times are i.i.d. In this model we classify the vacations into two types. The $V^{2 k-1}$ is the 1-policy vacation and it means the idle period during which the server waits for a customer. The $V^{2 k}$ is the ordinary vacation and it means the interval of the setup time. Clearly $l_{2 k-1}=0$ and $l_{2 k}=1$. In Figure $1, V^{2 k-1}$ is the interval $(a, b]$. The $(b, c]$ is the interval for setup time and so $V^{2 k}$ is this interval. The customer arriving at $b$ is the customer of 1-policy vacation $V^{2 k-1}$ and he is also the customer of $l_{2 k}=1$. He begins to receive service at $d$ because of LIFO discipline. In this model $J_{2 k-1}=(a, b] \cup(d, e]$ and $J_{2 k}=(b, d]$. That is, $J_{2 k-1}$ is interrupted by $J_{2 k}$. The stochastic behavior of $y_{t}$ on $J_{2 k-1}$ is independent of that on other time domain and it is equal to the stochastic behavior of a regenerative cycle of ordinary $M / G / 1$. Similarly the stochastic behavior of $y_{t}-l_{2 k}$ on $J_{2 k}$ is equal to the stochastic behavior of ordinary $M / G / 1$ with multiple vacation. So we will put $\xi_{2 k-1}=1$ and $\xi_{2 k}=2$ in later sections.

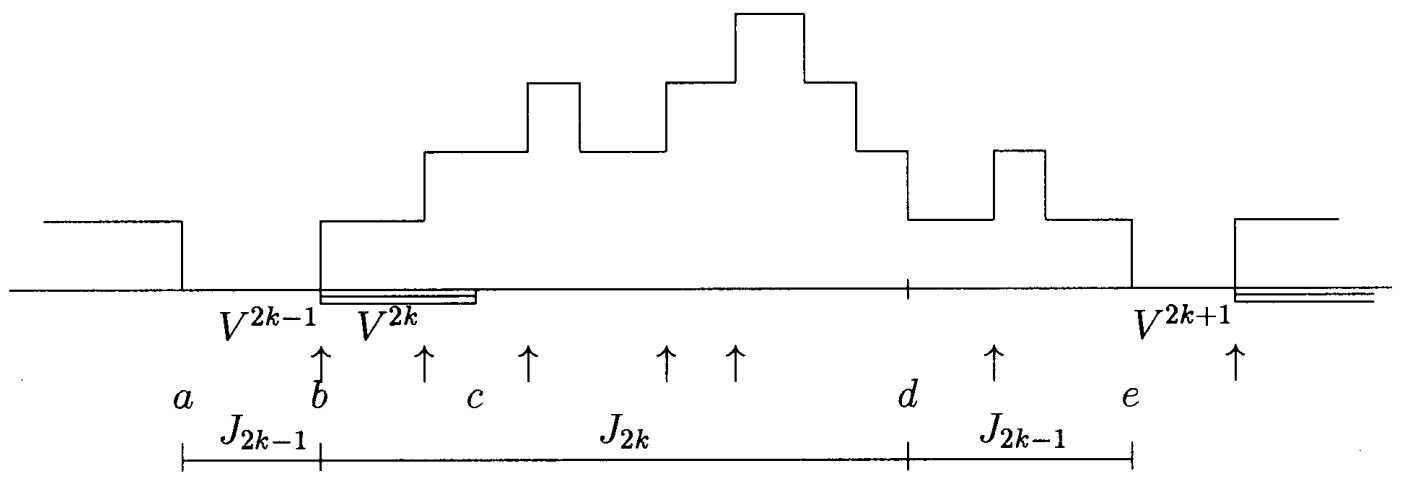

Figure 1. Queue length $y_{t}$ in the model with setup time.

Example 2.2. Consider the $M / G / 1$ with the pure limited vacation rule. In this model, the server takes one ordinary vacation after each service. In Figure $2, V^{1}, V^{3}, \cdots, V^{7}$ are such ordinary vacations. The $V^{2}$ and $V^{8}$ are 1-policy vacations. The server waits for next customer after returning from vacation $V^{1}$. The customer arriving at $b$ begins to receive the service at once, its service ends at $d$ and the server takes the vacation $V^{3}$. From LIFO discipline the customer arriving at $c$ begins to receive the service at $e$. The time domain generated by 1-policy vacation $V^{2}$ is $(a, d] \cup(e, f]$. Therefore the behavior of $y_{t}$ on the interval $(a, g]$ is the regenerative cycle of $M / G / 1$ which is interrupted by two intervals $(d, e]$ and $(f, g]$.

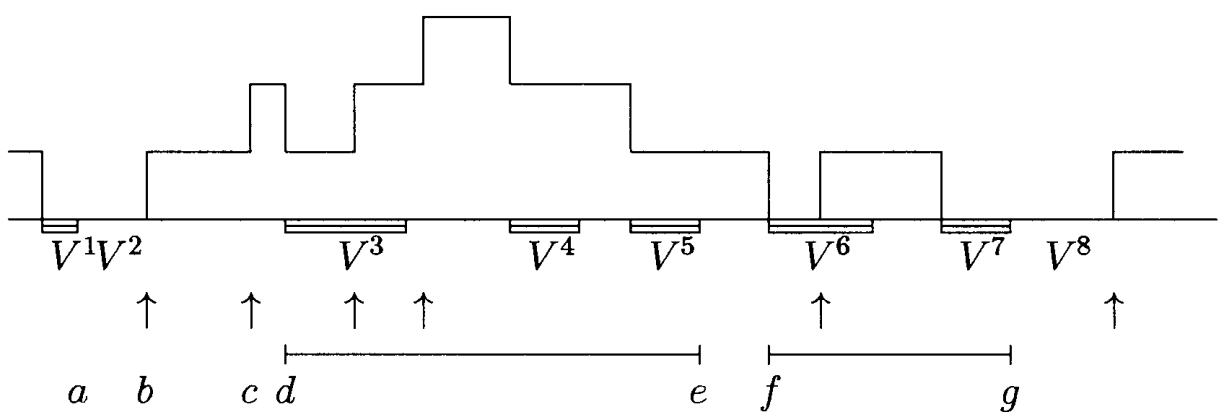

Figure 2. Queue length $y_{t}$ in $M / G / 1$ with pure limited vacation rule. 


\section{Time Average}

\subsection{Time average and distribution at an arbitrary time}

This paper considers the time average of the queue length and does not directly deal with the distribution at an arbitrary time for the following reason. The $M / G / 1$ with multiple vacation is the fundamentally important model in this paper. However the queue length in this model has no limiting distribution in some special cases. For example, let's consider the case that both the service time and the vacation time take the values of integer with probability one. If the first vacation begins at an epoch of integer, e.g., 0, this vacation ends at an epoch of integer. Since any time epoch belongs to the vacation period or the service period, all vacation times and all service periods begin at epochs of integer in spite of the arrival process. Conversely if the first vacation begins at an epoch of non-integer, all vacation times begin at epochs of non-integer. That is, the influence of the fixed initial state is eternal. Moreover if the first vacation begins at an epoch of integer, the customers leave the system only at epochs of integer, so that the distribution of queue length immediately before an integer is different from that immediately after this integer epoch. Thereby there is not the limiting distribution of the number of customers on the continuous time parameter domain for a fixed initial condition.

The beginning of the vacation in $M / G / 1$ with multiple vacation is the regeneration point. In above example the differences between these points are integers. That is, the renewal interval has the lattice distribution to which much of the regenerative theory is not applicable(see Asmussen[1]). On the other hand any regenerative process becomes stationary if we add the special initial condition(see [25, p.110]). From this fact most authors(e.g., $[7,9,12,19])$ used the term "distribution at an arbitrary time". We must take cares. This term does not imply the stability in the sense of the existence of the limiting distribution with a fixed initial state. Our result holds also for the model with the fixed initial state or even for non-regenerative process, so that we use the term "time average". As is well known, if a process is stationary and ergodic, or if it is the stationary regenerative process, its distribution at an arbitrary time is identical to the time average.

\subsection{Time average and regenerative process}

Let $(\Omega, \sigma(\Omega), P)$ be the basic probability space. Let $y_{t}$ be the nonnegative integer-valued process which we want to analyze. We use abbreviation w.p.1 for "with probability one in $P$ ". We define the time average of the set $u(\subset\{0,1,2, \cdots\})$ by

$$
T A(u)=\lim _{T \rightarrow \infty} T^{-1} \int_{0}^{T} \chi\left(y_{t} \in u\right) d t \quad \text { w.p.1 }
$$

if the limit of the right hand side exists, where $\chi(A)=1$ if $A$ holds and $\chi(A)=0$ otherwise. Note that this is generally not the probability of $u$ at an arbitrary time. Moreover (3.1) does not guarantee even the uniqueness. That is, it may depend on $\omega \in \Omega$. When the $T A(u)$ has the property of probability measure, its probability generating function(PGF) is defined as

$$
\Pi(z)=\sum_{j=0}^{\infty} T A(\{j\}) z^{j} .
$$

In this paper we call this the time average PGF of $y_{t}$ or simply the PGF of the model. If we get $\Pi(z)$, we can get $T A(\{n\})$ and the moments by differentiating $\Pi(z)$.

In the case that $y_{t}$ is the classical regenerative process with the finite mean of the cycle length, the time average of $y_{t}$ exists uniquely for every $u$. Let $(0 \leq) c_{1}<c_{2}<\cdots$ be the 
regeneration points. We define

$$
D_{k}=\int_{c_{k}}^{c_{k+1}} \chi\left(y_{t} \in u\right) d t \quad: k=1,2, \cdots
$$

Then the vector of two variables $\left\{D_{k}, c_{k+1}-c_{k}\right\}$ is identically independently distributed for every $u$. It is well known $([1,25])$ that the $T A(u)$ has the property of the probability measure and is equivalent to the distribution on a cycle, i.e.,

$$
T A(u)=\frac{E\left(D_{1}\right)}{E\left(c_{2}-c_{1}\right)}
$$

where $E(\bullet)$ represents the mean of the variable $\bullet$.

\subsection{Vacation model}

The examples of this paper deal with two kinds of vacations, the $N$-policy vacation and the ordinary vacation. We use the PGF's generated by these vacations without proof. They have the following forms. If, under the assumptions in section 2 the $J_{k}$ generated by the $N$-policy vacation is repeated independently and without interruption, it results in the regenerative process of the ordinary $M / G / 1$ with $N$-policy. We represent its time average PGF by $\Pi\left(z: M / G / 1 / N_{\text {policy }}\right)$. Particularly $\Pi\left(z: M / G / 1 / 1_{\text {policy }}\right)$ is equal to the well known $\operatorname{PGF} \Pi(z: M / G / 1)$. These PGF's have the following forms.

$$
\begin{aligned}
& \Pi\left(z: M / G / 1 / N_{\text {policy }}\right)=\frac{1-z^{N}}{N(1-z)} \Pi(z: M / G / 1) \\
& \Pi(z: M / G / 1)=\frac{(1-\rho)(1-z) B^{*}(\lambda-\lambda z)}{B^{*}(\lambda-\lambda z)-z}
\end{aligned}
$$

(see [20]). Let $\Theta_{k}$ be the length of $J_{k}$. Then $E\left(\Theta_{k}\right)=N /\{\lambda(1-\rho)\}$ is known for $N$-policy vacation.

In the case of the ordinary vacation with the distribution function $V(x)$, we denote the mean and LST of $V(x)$ by $v$ and $V^{*}(s)$ respectively. If the $J_{k}$ generated by the ordinary vacation repeats independently, it becomes $M / G / 1$ with multiple vacation. We represent this time average PGF by $\Pi(z: M / G / 1 / M V(V))$. As is well known, this is represented by

$$
\Pi(z: M / G / 1 / M V(V))=\frac{1-V^{*}(\lambda-\lambda z)}{\lambda v(1-z)} \Pi(z: M / G / 1) .
$$

Note that this model has two kinds of regeneration points. One is to choose all beginnings of vacations and other is to choose the epoch when the customer leaves no customer behind. This paper does not use the latter regeneration points. Therefore our $J_{k}$ in multiple vacation model has no service period, if no customer arrives during this vacation period, and it has the mean $E\left(\Theta_{k}\right)=v /(1-\rho)$.

\section{Theorems For Combination}

\subsection{General formation}

We assume that we can find a sequence of the time domains $\left\{J_{k}: k=1,2, \cdots\right\}$ with probability one in the model which we want to analyze. We discuss generally. So, besides the time domain generated by one vacation, we can choose other time domain as $J_{k}$. For example, we will choose $(d, e]$ in Example 2.2 as $J_{k}$ in later section. The $J_{k}$ satisfies the 
following conditions. First, $J_{k}$ has the starting epoch $t_{k}$. However $t_{k} \notin J_{k}$. For convenience we consider that the null set $J_{k}$ is possible, so that, if $J_{k}$ is not a null set, there is an open interval such that $\left(t_{k}, t_{k}+\epsilon\right) \subset J_{k}$. Secondly, $J_{k}$ has the finite end epoch $e_{k}$, i.e., $J_{k} \subset\left(t_{k}, e_{k}\right]$. The $e_{k}$ is contained in $J_{k}$ unless $J_{k}$ is a null set. Thirdly, $t_{i} \leq t_{j}$ if $i<j$. When $t_{i}=t_{j}(i<j)$, the $J_{i}$ is a null set. Fourthly, the $J_{i} \cap J_{j}$ is the null set if $i \neq j$. Fifthly, if $J_{i}$ is interrupted by $J_{j}$, then $J_{j}$ is not interrupted by $J_{i}$.

We assume that the model has the right-continuous nonnegative integer-valued process $y_{t}$. Let $\Theta_{k}$ be the length of $J_{k}$. Each $J_{k}$ is classified by its type number $\xi_{k}$. Let $\Xi$ be the space of values of $\xi_{k}$. We put $l_{k}=y_{t_{k}}$. Let $n_{k}(t) \equiv y_{t}-l_{k} \geq 0$ for $t \in J_{k}$. Since $J_{k}$ may be interrupted by other interval, we put $\tilde{n}_{k}(s)=n_{k}(t)$ for the length $s$ of $J_{k}$ on $(0, t]$. The following is our essential assumption.

Assumption 4.1. The number of elements in $\Xi$ is finite. The type $\xi_{k}$ is determined at $t_{k}$. Once $\xi_{k}$ is determined, the $\left(\Theta_{k}, \tilde{n}_{k}(s)\right)$ is distributed independently of past $y_{t}$ and past input containing arrival epochs, service times, vacation times and other variables. Its distribution is determined by the type number $\xi_{k}$. The $\Theta_{k}$ has the positive finite mean $\theta_{\xi}=E\left(\Theta_{k} \mid \xi_{k}=\xi\right)$.

In this assumption, first we should note that the value of $\xi_{k}$ is able to depend on the past $y_{t}$ or past input. Secondly although $\theta_{\xi}$ must be positive, the occurrence of $\Theta_{k}=0$ with positive probability is possible. The $J_{k}$ with $\Theta_{k}=0$ is a null set.

Thirdly when $\xi_{k}=\xi$ is given, we can define the probability measure $p_{i}^{\xi}$ by

$$
p_{i}^{\xi}=\theta_{\xi}^{-1} E\left(\int_{J_{k}} \chi\left(n_{k}(t)=i\right) d t \mid \xi_{k}=\xi\right)
$$

under the condition of $\xi_{k}=\xi$. We put

$$
\Pi(z: \xi)=\sum_{i=0}^{\infty} p_{i}^{\xi} z^{i}
$$

Since (4.1) is essentially identical to the distribution on $J_{k}$, from (3.4) the $\Pi(z: \xi)$ is the time average PGF of the regenerative process repeating $\tilde{n}_{k}(t)\left(\xi_{k}=\xi\right)$ independently.

Fourthly, since $\min \theta_{\xi}>0$ and each $J_{k}$ has the finite end epoch, one $J_{k}$ is not interrupted by the infinitely many other $J_{i}$ 's and $\left\{t_{k}\right\}$ has no accumulation epoch. Let $q$ be the maximum number such that $J_{k} \subset[0, T]$ for all $k \leq q$. Then we have $q \rightarrow \infty$ w.p. 1 as $T \rightarrow \infty$. We put $\epsilon_{T}=T-\sum_{k=1}^{q} \Theta_{k}$ and add the following two assumptions.

Assumption 4.2. $\cup_{k=1}^{\infty} J_{k}=(0, \infty)$.

Assumption 4.3. $\epsilon_{T} / T \rightarrow 0$ w.p. 1 as $T \rightarrow \infty$.

\subsection{Main theorem}

We will show the condition for the existence of $T A(j)$ under the above formation. Moreover we show that $T A(j)$ has the linear representation of $\Pi(z: \xi)$. For $q$ in Assumption 4.3, let $n_{q}^{\xi}$ be the number of $k$ such that $\xi_{k}=\xi$ and $k \leq q$. Let $n_{q}^{\xi}(i)$ be the number of $k$ such that $\xi_{k}=\xi, l_{k}=i$ and $k \leq q$. In the following assumption it is unnecessary that the limits $\alpha_{\xi}$ and $p_{i}^{l}(\xi)$ are constants with probability one.

Assumption 4.4. There is a finite limit $\alpha_{\xi}=\lim _{T \rightarrow \infty} n_{q}^{\xi} / T$ w.p.1 for each $\xi$. In addition, on the set $\left\{\omega: \alpha_{\xi}>0\right\}$ except for the set with probability 0 , there are numbers $p_{i}^{l}(\xi)$ such that $p_{i}^{l}(\xi)=\lim _{q \rightarrow \infty} n_{q}^{\xi}(i) / n_{q}^{\xi}$ and that $\sum_{i=0}^{\infty} p_{i}^{l}(\xi)=1$ holds. For other $\omega \in \Omega$ we put $p_{0}^{l}(\xi)=1$ and $p_{i}^{l}(\xi)=0$ for $i \geq 1$ in convenience. 
The $\alpha_{\xi}$ may not be unique. That is, it may depend on $\omega$, so that the following theorem does not guarantee the uniqueness of the value of $T A(j)$ or $\Pi(z)$. We put $\Pi^{l}(z: \xi)=$ $\sum_{i=0}^{\infty} p_{i}^{l}(\xi) z^{i}$

Theorem 4.1. Under Assumptions 4.1, 4.2, 4.3 and 4.4, the $T A(j)$ exists with probability one and $\{T A(j): j=0,1, \cdots\}$ constitutes a probability measure. Its $\operatorname{PGF} \Pi(z)$ is computed by

$$
\Pi(z) \equiv \sum_{j=0}^{\infty} T A(j) z^{j}=\sum_{\xi \in \Xi} \alpha_{\xi} \theta_{\xi} \Pi^{l}(z: \xi) \Pi(z: \xi)
$$

Proof. From Assumptions 4.2 and 4.3 we have

$$
\begin{aligned}
T A(j) & =\lim _{T \rightarrow \infty} \frac{1}{T} \int_{0}^{T} \chi\left(y_{t}=j\right) d t \\
& =\lim _{T \rightarrow \infty} \frac{1}{T} \sum_{k=1}^{q} \int_{J_{k}} \chi\left(y_{t}=j\right) d t \\
& =\lim _{T \rightarrow \infty} \frac{1}{T} \sum_{\xi \in \Xi} \sum_{i=0}^{j} \sum_{k=1}^{q} \chi\left(\xi_{k}=\xi, l_{k}=i\right) \int_{J_{k}} \chi\left(n_{k}(t)=j-i\right) d t
\end{aligned}
$$

Thus, from Assumptions 4.1 and 4.4 we get

$$
\begin{aligned}
T A(j) & =\lim _{T \rightarrow \infty} \sum_{\xi \in \Xi} \sum_{i=0}^{j} \frac{n_{q}^{\xi}}{T} \frac{n_{q}^{\xi}(i)}{n_{q}^{\xi}} \frac{1}{n_{q}^{\xi}(i)} \sum_{k=1}^{q} \chi\left(\xi_{k}=\xi, l_{k}=i\right) \int_{J_{k}} \chi\left(n_{k}(t)=j-i\right) d t \\
& =\sum_{\xi \in \Xi} \alpha_{\xi} \theta_{\xi} \sum_{i=0}^{j} p_{i}^{l}(\xi) p_{j-i}^{\xi} .
\end{aligned}
$$

Hence (4.3) follows.

Finally we must show $\sum_{i=0}^{\infty} T A(i)=1$. Since $\epsilon_{T}=T-\sum_{k=1}^{q} \Theta_{k}$, we have

$$
1=\lim _{T \rightarrow \infty} \frac{1}{T} \sum_{k=1}^{q} \Theta_{k}=\lim _{T \rightarrow \infty} \frac{1}{T} \sum_{\xi \in \Xi} \sum_{k=1}^{q} \Theta_{k} \chi\left(\xi_{k}=\xi\right)=\sum_{\xi \in \Xi} \alpha_{\xi} \theta_{\xi} .
$$

By putting $z=1$ in (4.3) we find $\sum_{\xi \in \Xi} \alpha_{\xi} \theta_{\xi}=\sum_{i=0}^{\infty} T A(i)$. Hence theorem follows.

Remark 1: The equality $\sum \alpha_{\xi} \theta_{\xi}=1$ in the proof is often used for determining $\alpha_{\xi}$.

Remark 2: The $\alpha_{\xi}$ and the $p_{i}^{l}(\xi)$ in Assumption 4.4 are constants in every example in this paper. However this is not the necessary condition. For example, consider the model such that, if the first service time after the system starts is smaller than a certain value, the system becomes $M / G / 1$ and, if not, it becomes $M / G / 1$ with multiple vacation. Then $T A(j)$ exists but it depends on the first service time.

Remark 3: Fuhrmann and Cooper[7] stated about three-way decomposition

$$
\Pi(z)=H_{-}(z) \alpha_{-}(z) \Pi(z: M / G / 1)
$$

This is the case that $\xi$ in our theorem takes only one type of the ordinary vacation. In this case it is written by $\Pi(z)=\Pi^{l}(z: \xi) \Pi(z: \xi)=\Pi^{l}(z: \xi) \alpha_{-}(z) \Pi(z: M / G / 1)$. 


\subsection{Case of the regenerative process}

In all applications in this paper the $y_{t}$ itself is the regenerative process, so that the following theorem is useful and Theorem 4.1 holds under Assumptions 4.1 and 4.2.

Theorem 4.2. Assume that the $y_{t}$ is the regenerative process with finite mean $\theta_{y}$ of the cycle length and that $\epsilon_{T}=0$ at the regeneration point. Then, Assumption 4.3 holds. The $\alpha_{\xi}$ and the $p_{i}^{l}(\xi)$ are uniquely determined for each $\xi$ and

$$
\begin{aligned}
\alpha_{\xi} \theta_{y} & =E\left(\text { The NO of the event }\left\{\xi_{k}=\xi\right\}\right. \text { in one RC), } \\
p_{i}^{l}(\xi) & =\frac{E\left(\text { The NO of the event }\left\{\xi_{k}=\xi, l_{k}=i\right\} \text { in one RC }\right)}{E\left(\text { The NO of the event }\left\{\xi_{k}=\xi\right\} \text { in one RC }\right)},
\end{aligned}
$$

where NO and RC are the abbreviations of "number of occurrences" and "regenerative cycle of $y_{t}$ " respectively. Assumption 4.4 also holds.

Proof. Since $\theta_{y}$ is finite, Assumption 4.3 holds. Let $T_{1}<T_{2} \cdots$ be regeneration points. Let $m_{i}^{\xi}$ be the number of occurrences of $J_{k}$ with $\xi_{k}=\xi$ in the $i$ th regenerative cycle of $y_{t}$. This $m_{i}^{\xi}$ is identically independently distributed. Hence (4.4) follows from

$$
\alpha_{\xi}=\lim _{T \rightarrow \infty} \frac{1}{T} n_{q}^{\xi}=\lim _{j \rightarrow \infty} \frac{j}{T_{j+1}} \frac{1}{j} \sum_{i=1}^{j} m_{i}^{\xi} .
$$

Similarly $p_{i}^{l}(\xi)$ is constant and (4.5) holds. By the monotone convergence theorem we have $\sum_{i=0}^{\infty} p_{i}^{l}(\xi)=1$, so that Assumption 4.4 holds.

\section{Examples; Setup Time}

This paper takes up several examples with one class of Poisson arrivals with intensity $\lambda$. This section considers two models with setup. Let $V(x)$ and $v$ be the distribution function of the length of the setup time and its mean respectively.

In the ordinary setup model in Example 2.1, we choose 1 and 2 as the values of the type $\xi$. The $\xi=1$ represents the 1-policy vacation $V^{2 k-1}$ and $\xi=2$ represents the ordinary vacation $V^{2 k}$ which means setup time with $V(x)$. Then $\theta_{1}=\{\lambda(1-\rho)\}^{-1}$ and $\theta_{2}=v /(1-\rho)$. Clearly the queue length $y_{t}$ is the regenerative process. As the regeneration point we choose the epoch when the customer leaves no customer behind. One regenerative cycle consists of two time domains $J_{2 k-1}$ and $J_{2 k}$, where $J_{i}$ is the time domains generated by $V^{i}$. Clearly the mean $\theta_{y}$ of cycle length satisfies $\theta_{y}=\theta_{1}+\theta_{2}$, so that $\alpha_{\xi}$ and $p_{i}^{l}(\xi)$ of Assumption 4.4 are uniquely determined from Theorem 4.2. Clearly $\alpha_{1}=\alpha_{2}$. Since $\alpha_{1} \theta_{1}+\alpha_{2} \theta_{2}=1$, we get $\alpha_{1}=\alpha_{2}=\lambda(1-\rho) /(1+\lambda v)$. On the other hand there is no customer at the beginning of $J_{2 k-1}$, so that $p_{0}^{l}(1)=\operatorname{Pr}\left\{l_{k}=0 \mid \xi_{k}=1\right\}=1$. There is one customer at the beginning of $J_{2 k}$ so that $p_{1}^{l}(2)=\operatorname{Pr}\left\{l_{k}=1 \mid \xi_{k}=2\right\}=1$. From Theorem 4.1 the time average PGF of the queue length $y_{t}$ of this model is given by

$$
\Pi\left(z:_{/ S \text { etup }}^{M / G / 1}\right)=\frac{1}{1+\lambda v} \Pi(z: M / G / 1)+\frac{\lambda v}{1+\lambda v} z \Pi\left(z:_{/ M V(V)}^{M / G / 1}\right) .
$$

This is identical to the equation of [20]p.131.

The differential $\Pi^{(n)}\left(z:_{/ \text {Setup }}^{M / G / 1}\right)$ is easily obtained from (5.1), if we know $\Pi^{(n)}(z: M / G / 1)$ and $\Pi^{(n)}(z: M / G / 1)$ which are discussed in last section. Similar arguments about differentials hold in equations in this and later sections. 
In the same way we can extend the type $\xi=1$ to $N$-policy. Its result is

$$
\Pi\left(z:_{/ \text {Setup }}^{M / G / 1 / N_{\text {policy }}}\right)=\frac{N}{N+\lambda v} \Pi\left(z:_{/ N_{\text {policy }}}^{M / G / 1}\right)+\frac{\lambda v}{N+\lambda v} z^{N} \Pi\left(z:_{/ M V(V)}^{M / G / 1}\right)
$$

(also see [20]p.135). We get $\theta_{y}=\theta_{1}+\theta_{2}=(N+\lambda v) /\{\lambda(1-\rho)\}$.

Secondly let's consider the model with multiple vacation and setup time. If the server finds customers at the end of the vacation, he begins to serve them after the setup time. If not, he takes another vacation. We choose 1 and 2 as the values of $\xi$. Both are the types of the ordinary vacation. The $\xi=1$ means the vacation of real model. Let $V_{1}(x)$ be its distribution function. Let $v_{1}$ and $V_{1}^{*}(s)$ be its mean and LST respectively. Then $\theta_{1}=v_{1} /(1-\rho)$. The type $\xi=2$ means the setup time with $V(x)$. As the regeneration point we choose every beginning of the vacation of type $\xi=1$. Then $\theta_{y}<\theta_{1}+\theta_{2}<\infty$ so that Theorems 4.1 and 4.2 hold. We have $\alpha_{1}: \alpha_{2}=1: 1-V_{1}^{*}(\lambda)$. Since $\alpha_{1} \theta_{1}+\alpha_{2} \theta_{2}=1$, we get

$$
\alpha_{1}=\frac{1-\rho}{\beta} \text { and } \alpha_{2}=\frac{(1-\rho)\left(1-V_{1}^{*}(\lambda)\right)}{\beta}
$$

where $\beta=v_{1}+v\left(1-V_{1}^{*}(\lambda)\right)$. There is no customer at the beginning of the vacation with $\xi=1$, so that $\Pi^{l}(z: \xi=1)=1$. The $\Pi^{l}(z: \xi=2)$ is equal to the PGF of the number of the customers arriving during the vacation with $\xi=1$ on the condition that at least one customer arrives, so that $\Pi^{l}(z: \xi=2)=\left\{V_{1}^{*}(\lambda-\lambda z)-V_{1}^{*}(\lambda)\right\} /\left(1-V_{1}^{*}(\lambda)\right)$. Hence

$$
\Pi\left(z:_{/ \text {Setup }}^{\bullet / M V\left(V_{1}\right)}\right)=\frac{v_{1}}{\beta} \Pi\left(z:_{/ M V\left(V_{1}\right)}^{M / G / 1}\right)+\frac{v}{\beta}\left(V_{1}^{*}(\lambda-\lambda z)-V_{1}^{*}(\lambda)\right) \Pi\left(z:_{/ M V(V)}^{M / G / 1}\right),
$$

where $\bullet$ is the abbreviation of " $M / G / 1$ ". We get $\theta_{y}=\theta_{1}+\left\{1-V_{1}^{*}(\lambda)\right\} \theta_{2}=\beta /(1-\rho)$. By the further calculation of (5.3) we find that it is equal to $Q^{s}(z)$ of $[2$, p.280].

\section{Examples: Simple State Dependent Vacation}

To $M / G / 1 / N_{\text {policy }}$ and $M / G / 1$ with multiple vacation this section adds the condition that the server takes an ordinary vacation only when the number of waiting customers at the epoch of the completion of the service is one. That is, $l_{k}=1$ for this vacation. We denote this distribution function, its mean, its LST and its type number by $V(x), v, V^{*}(s)$ and $\xi=2$ respectively. If Theorem 4.1 holds, we can represent

$$
\Pi\left(z:_{/ l_{k}=1}^{\bullet / N_{\text {policy }}}\right)=\frac{N \alpha_{1}}{\lambda(1-\rho)} \Pi\left(z: / N_{\text {policy }}\right)+\frac{v \alpha_{2}}{1-\rho} z \Pi\left(z:_{/ M V(V)}^{M / G / 1}\right)
$$

for this variant of $M / G / 1 / N_{\text {policy }}$ and

$$
\Pi\left(z:_{/ / l_{k}=1}^{\bullet / M V\left(V_{1}\right)}\right)=\frac{v_{1} \alpha_{1}}{1-\rho} \Pi\left(z:_{/ M V\left(V_{1}\right)}^{M / G / 1}\right)+\frac{v \alpha_{2}}{1-\rho} z \Pi\left(z: \frac{M / G / 1}{M V(V)}\right)
$$

for this variant of $M / G / 1 / M V\left(V_{1}\right)$.

This section considers only (6.1) with $N=1$ for the space saving. The $\xi=1$ corresponds to the vacation of 1-policy. We consider two cases. First we assume that the server takes a vacation of $\xi=2$ only once during one interval such as $y_{t}>0$. As a regeneration point we choose the epoch when the customer leaves no customer behind. Then $\theta_{y}<\theta_{1}+\theta_{2}=$ $(1+\lambda v) /\{\lambda(1-\rho)\}<\infty$, so that $\alpha_{\xi}$ and $p_{i}^{l}(\xi)$ of Assumption 4.4 are uniquely determined from Theorem 4.2. The server takes the vacation of $\xi=2$, if at least one customer arrives 
during the first service time. So we have $\alpha_{1}: \alpha_{2}=1: 1-B^{*}(\lambda)$. From $\alpha_{1} \theta_{1}+\alpha_{2} \theta_{2}=1$, we get $\lambda^{-1} \alpha_{1}+v \alpha_{2}=1-\rho$. Hence

$$
\alpha_{1}=\frac{\lambda(1-\rho)}{\beta} \text { and } \alpha_{2}=\frac{\lambda(1-\rho)\left(1-B^{*}(\lambda)\right)}{\beta},
$$

where $\beta=1+\lambda v\left(1-B^{*}(\lambda)\right)$. Therefore

$$
\Pi\left(z::_{/ l_{k}=1}^{M / G / 1}\right)=\frac{1}{\beta} \Pi(z: M / G / 1)+\frac{\lambda v\left(1-B^{*}(\lambda)\right)}{\beta} z \Pi\left(z: \frac{M / G / 1}{M V(V)}\right) .
$$

Secondly we assume that the server always takes the vacation of $\xi=2$, if he finds exactly one waiting customer at the end of the service of the customer. The regenerative cycle consists of the $J_{k}$ of 1-policy vacation with $\xi=1$ and the time domains $J_{k+1}, J_{k+2}, \cdots$ generated by ordinary vacations with $\xi=2$. If a customer arrives during the interval from the beginning of the ordinary vacation and to the end of one service immediately after this vacation, the server takes next ordinary vacation. Hence the probability that the server takes $m$ vacations during one period such as $y_{t}>0$ is

$$
\left\{\begin{array}{cc}
B^{*}(\lambda) & \text { if } m=0 \\
\left\{1-B^{*}(\lambda)\right\} V^{*}(\lambda) B^{*}(\lambda)\left\{1-V^{*}(\lambda) B^{*}(\lambda)\right\}^{m-1} & \text { if } m>0
\end{array}\right.
$$

The mean of $m$ is calculated as

$$
E(m)=\frac{1-B^{*}(\lambda)}{V^{*}(\lambda) B^{*}(\lambda)}
$$

Therefore from Wald's equation(e.g., [25]p.98) we find $\theta_{y}=\theta_{1}+E(m) \theta_{2}<\infty$. We have $\alpha_{1}: \alpha_{2}=1: E(m)$. Since $\lambda^{-1} \alpha_{1}+v \alpha_{2}=1-\rho$, we get

$$
\alpha_{1}=\frac{\lambda(1-\rho)}{1+\lambda v E(m)} \text { and } \alpha_{2}=\frac{\lambda(1-\rho) E(m)}{1+\lambda v E(m)} \text {. }
$$

Hence

$$
\Pi\left(z:_{/ l_{k}=1}^{M / G / 1}\right)=\frac{1}{1+\lambda v E(m)} \Pi(z: M / G / 1)+\frac{\lambda v E(m)}{1+\lambda v E(m)} z \Pi\left(z:_{/ M V(V)}^{M / G / 1}\right)
$$

\section{Base Model Interrupted By Regenerative Cycles}

The regenerative cycle in Example 2.1 begins with a vacation of 1-policy and the $J_{k}$ generated by this vacation is interrupted by one ordinary vacation. Similar interruption is seen in any model in sections 5 and 6 . Moreover in Example 2.2 the $J_{k}$ with 1-policy vacation is interrupted by the interval $(d, e]$ and followed by $(f, g]$. The probability structures on these intervals are the same if we ignore $y_{d}$ and $y_{f}$. Roughly speaking, in these models the well known regenerative process which we call the base model is interrupted by other regenerative cycles and resumes without sustaining any damage to its probability structure. The $y_{t}$ in these models is also a regenerative process whose regeneration point is the beginning of the cycle of the base model. If we give the interrupting regenerative cycle the type number $\xi$, this style has the PGF with the following form from Theorem 4.1.

$$
\Pi(z)=\alpha_{b a s e} \theta_{b a s e} \Pi_{b a s e}(z)+\alpha_{\xi} \theta_{\xi} \Pi^{l}(z: \xi) \Pi(z: \xi) .
$$


We can construct many models whose PGF's are obtained by this equation, if we know $\Pi_{\text {base }}(z)$ and $\Pi(z: \xi)$. Moreover, by using (7.1) repeatedly, we can obtain the PGF's for many complicated models.

In the examples in previous sections, $\Pi(z: \xi)$ is the PGF (3.7) of $M / G / 1$ with multiple vacation. In this case we have

$$
\Pi(z)=\alpha_{b a s e} \theta_{b a s e} \Pi_{b a s e}(z)+\frac{v \alpha_{\xi}}{1-\rho} \Pi^{l}(z: \xi) \Pi\left(z:_{/ M V(V)}^{M / G / 1}\right) .
$$

Typical application of (7.2) is the single vacation. The server takes a vacation with $V(x)$ at the end of a regenerative cycle of the base model and the cycle of $M / G / 1 / M V(V)$ begins. This cycle is not interrupted by other interval. Here we assume that, if he finds a customer on return from the vacation, he begins services and takes the vacation again at the end of this busy period. If he finds no customer on return from the vacation, the new cycle of the base model begins. The PGF of this model is represented in the form of (7.2). In this case $\alpha_{\text {base }}: \alpha_{\xi}=1: V^{*}(\lambda) \sum_{i=0}^{\infty}(i+1)\left(1-V^{*}(\lambda)\right)^{i}=1: V^{*}(\lambda)^{-1}$ and $\Pi^{l}(z: \xi)=1$. Therefore $\alpha_{\text {base }}=(1-\rho) V^{*}(\lambda) /\left(\theta_{\text {base }}(1-\rho) V^{*}(\lambda)+v\right)$ and

$$
\Pi(z)=\frac{(1-\rho) V^{*}(\lambda) \theta_{b a s e}}{(1-\rho) V^{*}(\lambda) \theta_{b a s e}+v} \Pi_{b a s e}(z)+\frac{v}{(1-\rho) V^{*}(\lambda) \theta_{b a s e}+v} \Pi\left(z:_{/ M V(V)}^{M / G / 1}\right) .
$$

When $\Pi_{\text {base }}(z)=\Pi(z: M / G / 1)$, the (7.3) becomes the equation (4.3) of [5].

It is possible to extend (7.2) to the independent selection of ordinary vacations which have the different distribution functions $V_{1}(x), \cdots, V_{p}(x)$. Let $v_{i}$ be the mean of $V_{i}(x)$. Assume that, when the server takes a vacation, he chooses $V_{i}(x)$ with probability $\delta_{i}$ and takes a corresponding vacation. If this selection is i.i.d. and independent of other variables, the PGF of the queue length at the beginning of the vacation is the same among these vacations so that we denote it by $\Pi^{l}(z: \xi=0)$. Then we have the following form from Theorem 4.1.

$$
\Pi(z)=\alpha_{b a s e} \theta_{b a s e} \Pi_{b a s e}(z)+\alpha_{0} \Pi^{l}(z: \xi=0) \sum_{i=1}^{p} \frac{\delta_{i} v_{i}}{1-\rho} \Pi\left(z:_{/ M V\left(V_{i}\right)}^{M / G / 1}\right) .
$$

The case of $v_{p}=0$ is possible in (7.4) or Theorem 4.1, so that we can deal with the Bernoulli schedule where the server takes a vacation with a certain probability $\delta$ (see $[20$, p.246]).

\section{Examples: Pure Limited Service System}

This section considers the model where the server takes an ordinary vacation at each end of the service, even if there is no customer at this epoch. Such model is called the pure limited service system $([11,20])$. Let $V(x)$ be the distribution function of the length of this ordinary vacation. Let $v$ and $V^{*}(s)$ be its mean and LST. As the base model we choose $M / G / 1, M / G / 1 / N_{\text {policy }}, M / G / 1$ with setup time, $M / G / 1 / M V\left(V_{1}\right)$ and so on. We denote these variants by $M / G / 1 / N_{\text {policy }} / P L(V), M / G / 1 / M V\left(V_{1}\right) / P L(V)$ and so on.

First we will consider $M / G / 1 / M V\left(V_{1}\right) / P L(V)$. We can write

$$
\Pi\left(z: \frac{M / G / 1}{M V\left(V_{1}\right) / P L(V)}\right)=\frac{\alpha_{1} v_{1}}{1-\rho} \Pi\left(z:_{/ M V\left(V_{1}\right)}^{M / G / 1}\right)+\frac{\alpha_{2} v}{1-\rho} \Pi^{l}(z) \Pi\left(z:_{/ M V(V)}^{M / G / 1}\right) .
$$

One vacation $V$ accompanies one service, so that $\alpha_{2}=\lambda$. Since $\left(\alpha_{1} v_{1}+\alpha_{2} v\right) /(1-\rho)=1$, we have $\alpha_{1}=(1-\rho-\lambda v) / v_{1}$. We will obtain $\Pi^{l}(z)$. Let $l_{k}$ be the number of customers at 
the beginning of the vacation of $V$ and let $a_{k}$ be the number of customers arriving during this vacation. Then $l_{k}+a_{k}$ is stochastically equal to the number of customers immediately after the service in $M / G(B * V) / 1 / M V\left(V_{1}\right)$. Therefore

$$
\Pi^{l}(z)=\Pi\left(z:_{/ M V\left(V_{1}\right)}^{M / G(B * V) / 1}\right) / V^{*}(\lambda-\lambda z)
$$

Consequently we get

$$
\begin{aligned}
\Pi\left(z:_{/ M V\left(V_{1}\right) / P L(V)}^{M / G / 1}\right)= & \frac{1-\rho-\lambda v}{1-\rho} \Pi\left(z:_{/ M V\left(V_{1}\right)}^{M / G / 1}\right) \\
& \quad+\frac{\lambda v}{(1-\rho) V^{*}(\lambda-\lambda z)} \Pi\left(z:_{/ M V\left(V_{1}\right)}^{M / G(B * V) / 1}\right) \Pi\left(z:_{/ M V(V)}^{M / G / 1}\right),
\end{aligned}
$$

where

$$
\Pi\left(z::_{/ M V(V)}^{M / G(B * V) / 1}\right)=\frac{(1-\rho-\lambda v)\left\{1-V^{*}(\lambda-\lambda z)\right\} B^{*}(\lambda-\lambda z) V^{*}(\lambda-\lambda z)}{\lambda z\left\{B^{*}(\lambda-\lambda z) V^{*}(\lambda-\lambda z)-z\right\}}
$$

from the equation (4.3).

Particularly when $V_{1}=V$, we put

$$
\begin{aligned}
\Pi\left(z: \xi^{P L}\right) & \equiv \Pi\left(z:_{/ M V(V) / P L(V)}^{M / G / 1}\right) \\
& =\frac{(1-\rho-\lambda v)\left\{B^{*}(\lambda-\lambda z)-z\right\}}{(1-\rho)\left\{B^{*}(\lambda-\lambda z) V^{*}(\lambda-\lambda z)-z\right\}} \Pi(z: M / G / 1 / M V(V)) \\
& =\frac{(1-\rho-\lambda v) B^{*}(\lambda-\lambda z)\left(1-V^{*}(\lambda-\lambda z)\right)}{\lambda v\left\{B^{*}(\lambda-\lambda z) V^{*}(\lambda-\lambda z)-z\right\}} .
\end{aligned}
$$

This is equal to the equation calculated from [20, (6.103)].

We will try to use (7.1) by inserting (8.1) to other base model. Let's note that, since the model has the nonpreemptive LIFO discipline, the length of the interval from the end of the service of the base model to the epoch at which the base model begins again, e.g., the interval $(d, e]$ or $(f, g)$ in Example 2.2, is stochastically identical. That is, it is stochastically equal to the length of the regenerative cycle in $M / G(B * V) / 1 / M V(V)$, so that its mean is $v /(1-\rho-\lambda v)$. We choose this interval as one $J_{k}$. The probability structure of $y_{t}-l_{k}$ on this interval is equal to a regenerative cycle of $M / G / 1 / M V(V)$ with pure limited vacation rule, i.e., $M / G / 1 / M V(V) / P L(V)$ of (8.1). We give this interval the type number $\xi^{P L}$.

Let's consider the model in which a base model is interrupted by the $J_{k}$ with type $\xi^{P L}$. Let $\Pi_{b a s e}(z)$ and $\theta_{\text {base }}$ be the PGF of the base model and the mean of its cycle length respectively. Since the mean of the number of customers arriving during a regenerative cycle in the base model is $\lambda \theta_{\text {base }}$, the mean $\theta_{y}$ of the regeneration cycle of $y_{t}$, e.g., $(a, g]$ in Example 2.2 , is given by

$$
\theta_{y}=\theta_{b a s e}+\lambda \theta_{b a s e} \frac{v}{1-\rho-\lambda v}=\frac{1-\rho}{1-\rho-\lambda v} \theta_{b a s e}
$$

so that we assume $\rho+\lambda v<1$. Hence the (7.1) is written in the form:

$$
\Pi(z: / P L(V))=\alpha_{b a s e} \theta_{b a s e} \Pi_{b a s e}(z)+\alpha_{P L} \frac{v}{1-\rho-\lambda v} \Pi^{l}\left(z: \xi^{P L}\right) \Pi\left(z: \xi^{P L}\right) .
$$


Since $\alpha_{b a s e}: \alpha_{P L}=1: \lambda \theta_{b a s e}$ and $\alpha_{b a s e} \theta_{b a s e}+\alpha_{P L} v /(1-\rho-\lambda v)=1$, we have

$$
\alpha_{b a s e} \theta_{b a s e}=\frac{1-\rho-\lambda v}{1-\rho} \quad \text { and } \quad \alpha_{P L}=\lambda \frac{1-\rho-\lambda v}{1-\rho} .
$$

The regenerative cycle $\xi^{P L}$ begins at every end of service of the base model. Therefore $\Pi^{l}\left(z: \xi^{P L}\right)$ is the distribution of the queue length immediately after the departure epoch in the base model. From Burke's theorem and Wolff's PASTA, we have $\Pi^{l}\left(z: \xi^{P L}\right)=\Pi_{b a s e}(z)$. Hence (8.2) becomes

$$
\begin{aligned}
\Pi(z) & =\left(\frac{1-\rho-\lambda v}{1-\rho}+\frac{\lambda v}{1-\rho} \Pi\left(z: \xi^{P L}\right)\right) \Pi_{b a s e}(z) \\
& =\frac{(1-\rho-\lambda v)\left\{B^{*}(\lambda-\lambda z)-z\right\}}{(1-\rho)\left\{B^{*}(\lambda-\lambda z) V^{*}(\lambda-\lambda z)-z\right\}} \Pi_{b a s e}(z) .
\end{aligned}
$$

For example

$$
\Pi(z: M / G / 1 / P L(V))=\frac{(1-\rho-\lambda v)(1-z) B^{*}(\lambda-\lambda z)}{B^{*}(\lambda-\lambda z) V^{*}(\lambda-\lambda z)-z}
$$

\section{Examples: Decrementing Service System}

Takagi[20], Bischof[2] etc. discussed the decrementing service system where the server memorizes the queue length at the end of the vacation. In this section we assume that the server observes the system at the beginning of the vacation. That is, when the ordinary vacation $V^{k}$ of this rule starts, the server memorizes the value $l_{k}(>0)$ and takes a rest. Returning from that vacation, he continues to work until the number of customers decreases to $l_{k}-1$ and takes another vacation $V^{k+1}$. This is repeated until there is no customer in the system. Assume that these vacations have the same distribution function $V(x)$ with mean $v$ and LST $V^{*}(s)$. We give them the type number $\xi^{V}$. If we consider such model as the variation of the base model, its PGF has the form:

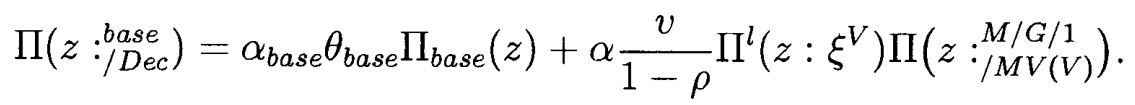

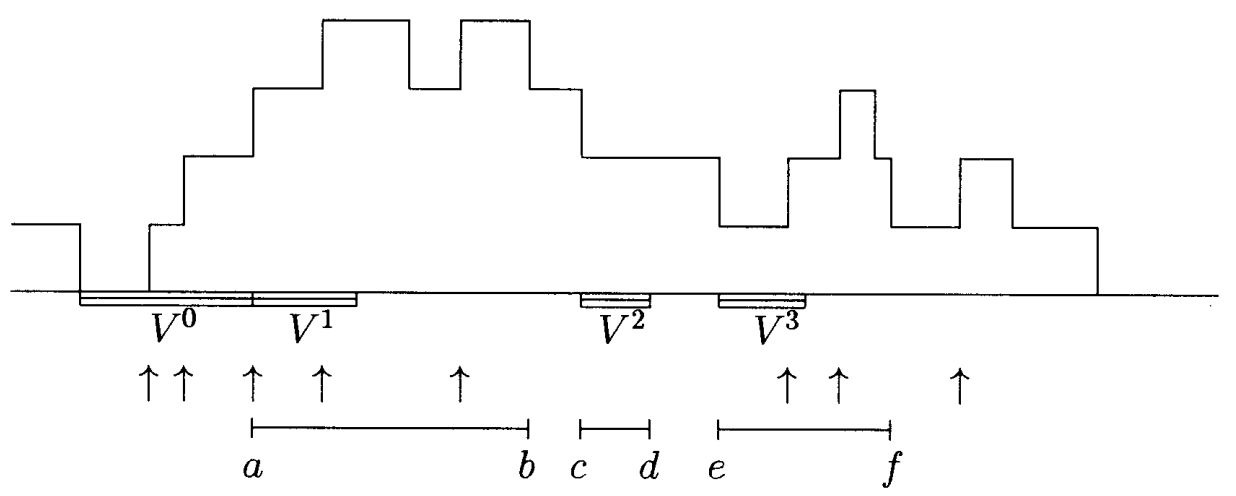

Figure 3. Queue length $y_{t}$ in $M / G / 1 / 3_{\text {policy }}$ with decrementing service rule.

First we choose $M / G / 1 / N_{\text {policy }}$ as the base model. We assume that the server takes the first vacation of type $\xi^{V}$ when the $N$ th customer in $N$-policy vacation arrives. Figure 3 illustrates the case of $N=3$. The $V^{0}$ is the 3 -policy vacation. The server takes the 
first vacation at $a$ and $(a, b)$ is the interval generated by this vacation. Similarly $(c, d)$ and $(e, f)$ are the intervals generated by the second and the third vacations respectively. The probability structure of the remaining parts is equal to $M / G / 1 / N_{\text {policy }}$. Clearly $l_{1}=3, l_{2}=2$ and $l_{3}=1$.

After the $N$-policy vacation he takes $N$ vacations with $l_{k}=N, N-1, \cdots, 1$. The mean of the length of a regenerative cycle of $y_{t}$ is $\theta_{y}=N(1+\lambda v) /\{\lambda(1-\rho)\}<\infty$, so that Theorem 4.2 holds. We have $\alpha_{\text {base }}: \alpha=1: N, \theta_{\text {base }}=N /\{\lambda(1-\rho)\}$ and $\Pi^{l}\left(z: \xi^{V}\right)=N^{-1} \sum_{i=1}^{N} z^{i}$. From these equations we get

$$
\Pi\left(z:_{/ D e c}^{\bullet / N_{\text {policy }}}\right)=\frac{1}{1+\lambda v} \Pi\left(z:_{/ N_{\text {policy }}}^{M / G / 1}\right)+\frac{\lambda v}{N(1+\lambda v)} \frac{z-z^{N+1}}{1-z} \Pi\left(z:_{/ M V(V)}^{M / G / 1}\right) .
$$

Next we choose $M / G / 1 / M V\left(V_{1}\right)$ as the base model. When customers arrive during a vacation with $V_{1}(x)$, the server takes first vacation of type $\xi^{V}$ at the end of the vacation with $V_{1}(x)$ and takes other vacations according to the decrementing vacation rule. In (9.1) the ratio $\alpha_{\text {base }}: \alpha=1: \lambda v_{1}$ holds and $\alpha_{b a s e} v_{1} /(1-\rho)+\alpha v /(1-\rho)=1$. One and only one vacation of type $\xi^{V}$ with $l_{k}=j$ is generated in one regenerative cycle of $y_{t}$ if more than or equal to $j$ customers arrive during the vacation with $V_{1}(x)$. Let $p$ customers arrive during a vacation with $V_{1}(x)$. Then,

$$
\begin{aligned}
\Pi^{l}\left(z: \xi^{V}\right) & =\sum_{i=0}^{\infty} p_{i}^{l}\left(\xi^{V}\right) z^{i}=\frac{1}{\lambda v_{1}} \sum_{i=1}^{\infty} \operatorname{Pr}(p \geq i) z^{i} \\
& =\frac{1}{\lambda v_{1}} \sum_{i=1}^{\infty} \sum_{j=i}^{\infty} \operatorname{Pr}(p=i) z^{i} \\
& =\frac{z}{\lambda v_{1}(1-z)} \sum_{j=1}^{\infty}\left(1-z^{j}\right) \operatorname{Pr}(p=j) \\
& =\frac{z}{\lambda v_{1}(1-z)}\left(1-V_{1}^{*}(\lambda-\lambda z)\right) .
\end{aligned}
$$

Consequently

$$
\Pi\left(z: / D e c\left(V_{1}\right)\right)=\frac{1}{1+\lambda v} \Pi\left(z:_{/ M V\left(V_{1}\right)}^{M / G / 1}\right)+\frac{v z\left\{1-V_{1}^{*}(\lambda-\lambda z)\right\}}{(1+\lambda v) v_{1}(1-z)} \Pi(z: / M V / G(V)) .
$$

\section{Examples: Bernoulli Feedback}

The results in this paper can be easily extended to the model with Bernoulli feedback. In this model the customer joins the queue immediately after the completion of his each service with probability $1-\sigma$ and departs forever with probability $\sigma$. Let $B_{F}(x)$ be the distribution of the total service time of one customer. Then its $\operatorname{LST} B_{F}^{*}(s)$ is given by

$$
B_{F}^{*}(s)=\frac{\sigma B^{*}(s)}{1-(1-\sigma) B^{*}(s)}
$$

and the mean of $B_{F}(x)$ is $b / \sigma($ see $[20]$ p.50). In Bernoulli feedback the distribution of the queue length is the same among FIFO, nonpreemptive LIFO, service in random order etc. So we consider the case that the customer receives his all services without interruption by other customer and that the service discipline is the nonpreemptive LIFO. Therefore, 
unless other vacation interrupts the continuity of his services, we can extend the previous all examples to the feedback model by replacing $B(x)$ with $B_{F}(x)$.

Here we consider three variants of the Bernoulli feedback. First, we consider the case that, if there is no other customer at the end of a service of a customer, the server takes a rest until there exist $N(>1)$ customers in the system. That is, if that customer requires another service, he must wait for arrivals of other $N-1$ customers. If not, the server waits for $N$ new customers. We choose 1 and 2 as the values of $\xi$. The $\xi=1$ represents the $(N-1)$-policy vacation and the $\xi=2$ represents $N$-policy vacation. As the regeneration point of $y_{t}$ we choose the beginning of the vacation of $N$-policy. The type is determined by whether the last customer requires another service or not. If $H$ is the number of occurrences of the vacation of the $(N-1)$-policy in a regenerative cycle, its probability is given by $\operatorname{Pr}\{H=j\}=\sigma(1-\sigma)^{j}$. Hence the mean $\theta_{y}$ of cycle length is finite from Wald's equation. We have $\alpha_{1}: \alpha_{2}=1-\sigma: \sigma$. From $\theta_{1} \alpha_{1}+\theta_{2} \alpha_{2}=1$, we have $(N-1) \alpha_{1}+N \alpha_{2}=\lambda(1-\lambda b / \sigma)$. Hence we get

$$
\begin{gathered}
\Pi(z: \text { Feedback } 1)=\frac{(N-1)(1-\sigma)}{N-1+\sigma} z \Pi\left(z: M / G\left(B_{F}\right) / 1 /(N-1)_{\text {policy }}\right) \\
+\frac{N \sigma}{N-1+\sigma} \Pi\left(z: M / G\left(B_{F}\right) / 1 / N_{\text {policy }}\right) .
\end{gathered}
$$

Second, the customer receives only one service. However, if he can find no customer behind at the completion of his service, he receives another service with probability $1-\sigma$. The interval from the beginning of this additional service to the next service completion time where its customer finds no customer behind is stochastically equal to the busy period of $M / G / 1$. Its PGF $\Pi(z:$ busy $)$ satisfies

$$
\Pi(z: M / G / 1)=1-\rho+\rho \Pi(z: b u s y)
$$

which is the simple case of Theorem 4.1. Hence the PGF of our second variant of the original feedback system is written as

$$
\Pi(z: \text { Feedback } 2)=\alpha_{1} \theta_{\text {base }} \Pi_{\text {base }}(z)+\alpha_{2} \frac{b}{1-\rho} \Pi(z: \text { busy }) .
$$

When the base model is $M / G / 1$, we have $\alpha_{1}+\alpha_{2} \rho=\lambda(1-\rho)$ and

$$
\Pi(z: F e e d b a c k 2)=\frac{\alpha_{1}+\alpha_{2}}{\lambda(1-\rho)} \Pi(z: M / G / 1)-\frac{\alpha_{2}}{\lambda} .
$$

If there is no restriction on the number of feedbacks, then $\alpha_{1}: \alpha_{2}=1:(1-\sigma) / \sigma$. We have

$$
\Pi(z: \text { Feedback } 2)=\frac{1}{\sigma+(1-\sigma) \rho} \Pi(z: M / G / 1)-\frac{(1-\sigma)(1-\rho)}{\sigma+(1-\sigma) \rho} .
$$

Thirdly we will consider the pure limited service system with Bernoulli feedback. We assume that the sequence of services of one customer is not interrupted by the service of other customer. If a customer receives the $n$ services, he experiences $n-1$ vacations of the server with $V(x)$ from the beginning of his service to his departing epoch. The LST of the distribution of this time length is given by

$$
B_{\text {pure }}^{*}(s)=\frac{\sigma B^{*}(s)}{1-(1-\sigma) B^{*}(s) V^{*}(s)}
$$


with the mean $(b+v-\sigma v) / \sigma$. If we regard this as the service period, our model becomes the ordinary pure limited service system. Therefore by replacing $B^{*}(s)$ in (8.4) by $B_{\text {pure }}^{*}(s)$ we can obtain the PGF of the feedback model with the pure limited vacation rule for each corresponding base model.

Wortman et al.[24] considered the case that the server takes vacation $V$ with probability $\delta$ immediately after each service. Such vacation is equal to the vacation $V_{0}$ with the LST $V_{0}^{*}(s)=1-\delta+\delta V^{*}(s)$ and the mean $v_{0}=\delta v$. We substitute this into $V^{*}(s)$ of (10.4) and put

$$
B_{\delta}^{*}(s)=\frac{\sigma B^{*}(s)}{1-(1-\sigma) B^{*}(s)\left(1-\delta+\delta V^{*}(s)\right)} .
$$

While they do not write their model in detail, their model is considered to be our $\xi^{P L}$ from the matrix $D_{d v}$ of [24]. So we will substitute $B_{\delta}^{*}(s)$ to (8.4). Then we obtain their equation (10) with different coefficient. Their equation (10) does not satisfy $\lim _{z \rightarrow 1} \Psi_{d}(z)=1$.

\section{Appendix: Probabilities and Moments}

Differentiating PGF, we can obtain probabilities and moments of its probability measure. However to differentiate the equation of PGF directly is very laborious. This is the serious problem for us, because our final aim is not PGF but these values. The technique which the early authors showed were laborious or vaguely represented, so this section shows the easy recursive calculation.

\subsection{Calculation of probabilities}

The PGF $\Pi(z)$ appearing in this paper has the form $\Pi(z)=D(z) / C(z)$ where $C(1)=$ $D(1)=0$ and $C(0) \neq 0$. We write

$$
C(z) \Pi(z)=D(z)
$$

By differentiating both sides $n$ times, we get

$$
\sum_{i=0}^{n}\left(\begin{array}{c}
n \\
i
\end{array}\right) C^{(n-i)}(z) \Pi^{(i)}(z)=D^{(n)}(z) .
$$

Hence the following recursive relation holds.

$$
\Pi^{(n)}(0)=\frac{1}{C(0)}\left\{-\sum_{i=0}^{n-1}\left(\begin{array}{l}
n \\
i
\end{array}\right) C^{(n-i)}(0) \Pi^{(i)}(0)+D^{(n)}(0)\right\} .
$$

From this relation we can obtain the probability $p_{n}=\Pi^{(n)}(0) / n$ ! or time average of (3.1).

The (11.2) is useful for the complicated PGF like $\Pi\left(z: \xi^{P L}\right)$ of (8.1). In $M / G / 1$ some authors([4, p.214][8, p.232][13]) recommended to use the transition matrix of the Markov chain. If we use (11.2) in this model, we put $C(z)=B^{*}(\lambda-\lambda z)-z, D(z)=$ $(1-\rho)(1-z) B^{*}(\lambda-\lambda z)$ and $c_{n}=(-\lambda)^{n} B^{*(n)}(\lambda)$. Then $C^{\prime}(0)=c_{1}-1$ and $C^{(n)}(0)=c_{n}$, if $n \geq 2$. Moreover, $D^{(n)}(0)=(1-\rho)\left(c_{n}-n c_{n-1}\right)$. Since $\Pi(0)=1-\rho$, the $(11.2)$ is written as

$$
\begin{aligned}
\Pi^{(n)}(0) & =\frac{1}{B^{*}(\lambda)}\left\{-\sum_{i=0}^{n-1}\left(\begin{array}{c}
n \\
i
\end{array}\right) c_{n-i} \Pi^{(i)}(0)+n \Pi^{(n-1)}(0)+D^{(n)}(0)\right\} \\
& =\frac{1}{B^{*}(\lambda)}\left\{-\sum_{i=1}^{n-1}\left(\begin{array}{c}
n \\
i
\end{array}\right) c_{n-i} \Pi^{(i)}(0)+n \Pi^{(n-1)}(0)-n(1-\rho) c_{n-1}\right\} .
\end{aligned}
$$


From this we easily calculate $\Pi^{(n)}(0)$ by hand for small $n$ and by the computer for large $n$.

In $M / G / 1 / M V(V)$ we put

$$
\zeta(z)=\frac{1-V^{*}(\lambda-\lambda z)}{\lambda v(1-z)}
$$

Applying (11.2) to this function, we obtain

$$
\begin{aligned}
\zeta^{(n)}(0) & =n \zeta^{(n-1)}(0)+\frac{1}{v}(-\lambda)^{n-1} V^{*(n)}(\lambda) \\
& =\frac{n !}{\lambda v}\left\{1-\sum_{i=0}^{n} \frac{1}{i !}(-\lambda)^{i} V^{*(i)}(\lambda)\right\} .
\end{aligned}
$$

Hence

$$
\Pi^{(n)}\left(0:_{/ M V(V)}^{M / G / 1}\right)=\sum_{i=0}^{n}\left(\begin{array}{c}
n \\
i
\end{array}\right) \zeta^{(n-i)}(0) \Pi^{(i)}(0: M / G / 1)
$$

Most of the PGF's in this paper are represented by the simple combination of $\Pi(z$ : $\left.M / G / 1 / N_{\text {policy }}\right)$ and $\Pi(z: M / G / 1 / M V(V))$, so that we can obtain the probability of such PGF by combining $\Pi^{(n)}\left(0: M / G / 1 / N_{\text {policy }}\right)$ and $\Pi^{(n)}(0: M / G / 1 / M V(V))$.

\subsection{Calculation of moments}

We will consider the calculation for the factorial moments $\Pi^{(n)}(1)$. Standard textbooks ([10][20]) recommend the direct differentiation and to use the L'Hôpital's rule. Several authors $[18,21,22]$ used Taylor expansion for the moments of the waiting time. Its method can be applicable to the queue length. Neuts[14, p.17] also proposed other method. The author thinks that the following modification of Riordan[15, p.70] is most convenient.

By differentiating both sides of (11.1) $n+1$ times, we get

$$
\sum_{i=0}^{n+1}\left(\begin{array}{c}
n+1 \\
i
\end{array}\right) C^{(n+1-i)}(z) \Pi^{(i)}(z)=D^{(n+1)}(z) .
$$

We put $z=1$. Since $C(1)=0$, we have

$$
\begin{aligned}
& (n+1) C^{\prime}(1) \Pi^{(n)}(1)+\sum_{i=0}^{n-1}\left(\begin{array}{c}
n+1 \\
i
\end{array}\right) C^{(n+1-i)}(1) \Pi^{(i)}(1)=D^{(n+1)}(1), \\
& \Pi^{(n)}(1)=-\frac{1}{(n+1) C^{\prime}(1)}\left\{\sum_{i=0}^{n-1}\left(\begin{array}{c}
n+1 \\
i
\end{array}\right) C^{(n+1-i)}(1) \Pi^{(i)}(1)-D^{(n+1)}(1)\right\} .
\end{aligned}
$$

In $M / G / 1$ we get

$$
\Pi^{(n)}(1: M / G / 1)=\frac{1}{1-\rho} \sum_{i=0}^{n-1}\left(\begin{array}{c}
n \\
i
\end{array}\right) \frac{\lambda^{n-i+1} b_{n-i+1}}{n-i+1} \Pi^{(i)}(1: M / G / 1)+\lambda^{n} b_{n}
$$

where $b_{n}=(-1)^{n} B^{*(n)}(0)$. Using this recursive relation we can obtain $\Pi^{(n)}(1: M / G / 1)$ easily by the hand work for small $n$ and by the computer for large $n$. 
We can obtain $\Pi^{(n)}(1: M / G / 1 / M V)$ from the equation:

$$
\begin{aligned}
\Pi^{(n)}\left(1: \begin{array}{l}
M / G / 1 \\
/ M V(V)
\end{array}\right) & =\sum_{i=0}^{n}\left(\begin{array}{c}
n \\
i
\end{array}\right) \zeta^{(n-i)}(1) \Pi^{(i)}(1: M / G / 1) \\
& =\sum_{i=0}^{n}\left(\begin{array}{c}
n \\
i
\end{array}\right) \frac{(-\lambda)^{n-i} V^{*(n-i)}(0)}{n-i+1} \Pi^{(i)}(1: M / G / 1) .
\end{aligned}
$$

\section{Acknowledgements}

The author thanks two anonymous referees for kindful comments.

\section{References}

[1] S. Asmussen: Applied Probability and Queues(John Wiley \& Sons, New York, 1987).

[2] W. Bischof: Analysis of $M / G / 1$-queues with setup times and vacations under six different service disciplines. Queueing systems, 39 (2001) 265-301.

[3] R. B. Cooper: Queues served in cyclic order: waiting times. The Bell System Technical Journal, 49 (1970) 399-413.

[4] R. B. Cooper: Introduction to Queueing Theory(Elsevier Science Publishing Co., Inc, New York, 1981).

[5] B.T. Doshi: Queueing systems with vacations-a survey. Queueing systems, 1 (1986) 29-66.

[6] B.T. Doshi: Conditional and unconditional distributions for $M / G / 1$ type queues with server vacations. Queueing systems, 7 (1990) 229-252.

[7] S. W. Fuhrmann and R. B. Cooper: Stochastic decompositions in the $M / G / 1$ queues with generalized vacations. Operations Research, 33 (1985) 1117-1129.

[8] D. Gross and C. M. Harris: Fundamentals of Queueing Theory(John Wiley \& Sons, New York, 1974).

[9] O. Kella: The threshold policy in the $M / G / 1$ queue with server vacations. Naval Research Logistics, 36 (1989) 111-123.

[10] L. Kleinrock: Queueing Systems Volume 1:Theory(John Wiley \& Sons, New York, 1975).

[11] T. T. Lee: $M / G / 1 / N$ queue with vacation time and limited service discipline. Performance Evaluation, 9 (1989) 181-190.

[12] D. M. Lucantoni, K. S. Meier-Hellstern and M. F. Neuts: A single-server queue with server vacations and a class of non-renewal arrival processes. Advances Applied Probability, 22 (1990) 676-705.

[13] M. F. Neuts: Queues solvable without Rouche's theorem. Operations Research, 27 (1979) 767-781.

[14] M. F. Neuts: Structured Stochastic Matrices of $M / G / 1$ Type and their Applications(Marcel Dekker, Inc, New York, 1989).

[15] J. Riordan: Stochastic Service System(John Wiley \& Sons, New York, 1962).

[16] J. G. Shanthikumar: On stochastic decomposition in $M / G / 1$ type queues with generalized server vacations. Operations Research, 36 (1988) 566-569.

[17] S. Sumita: Performance analysis of interprocessor communications in an electronic switching system with distributed control. Performance Evaluation, 9 (1989) 83-91. 
[18] L. Takács: A single-server queue with Poisson input. Operations Research, 10 (1962) 388-394.

[19] H. Takagi: Time-dependent analysis of $M / G / 1$ vacation models with exhaustive service, Queueing Systems, 6 (1990) 369-390.

[20] H. Takagi: Queueing Analysis, Volume 1:Vacation and Priority Systems, Part 1(NorthHolland, New York, 1991).

[21] H. Takagi and K. Sakamaki: Symbolic moment calculation for an M/G/1 queue. Discussion Paper No.596, Institute of Socio-Economic Planning, University of Tsukuba, (1994) 1-27.

[22] H. Takagi and K. Sakamaki: Moments for M/G/1 queues. The Mathematica Journal, 6 (1996) 75-80.

[23] M. A. Wortman and R. L. Disney: Vacation queues with Markov schedules, Advances Applied Probability, 22 (1990) 730-748.

[24] M. A. Wortman, R. L. Disney and P.C.Kiessler: The $M / G / 1$ Bernoulli feedback queue with vacations, Queueing Systems, 9 (1991) 353-363.

[25] R. W. Wolff: Stochastic Modeling and the Theory of Queues(Prentice-Hall, Englewood Cliffs, 1989).

[26] Z. G. Zhang, R. G. Vickson and M. J. A. van Eenige: Optimal two-threshold policies in an $M / G / 1$ queue with two vacation types, Performance Evaluation, 29 (1997) 63-80.

Toshinao Nakatsuka:

Faculty of Economics

Tokyo Metropolitan University

1-1 Hachiouji-shi

Tokyo, 192-0397, Japan

E-mail: tnaka@bcomp.metro-u.ac.jp 